\title{
Calculation of Perturbations in the Stellar Three-Body Problem
}

\author{
J. A. DOCOBO, C. PRIETO, \& J. F. LING \\ Observatorio Astronómico Ramón M. Aller, Universidad de Santiago, \\ P.O. Box 197, Santiago de Compostela, Spain
}

\begin{abstract}
The method of perturbations of Deprit has been applied to the stellar three-body problem. The calculation, developed previously by the authors in other articles, is here systematicized for easy use and applied to various triple systems.
\end{abstract}

\section{INTRODUCTION}

The stellar three-body problem (Harrington 1968a,1969; Docobo 1977; Abad 1984; Docobo \& Prieto 1988; Ling 1989) is concerned with the motion of three comparable masses $m_{0}, m_{1}$ and $m_{2}$, such that $m_{2}$ lies far enough from the others to be able to take the motion of $m_{1}$ about $m_{0}$ and that of $m_{2}$ about the center of mass of $m_{0}$ and $m_{1}$ as perturbed Keplerian orbits.

This problem has lately been aproached in several ways: hypothesis of Keplerian outer orbit; study of the perturbations of the outer orbit; integration of the equations of motion in various systems of variables; use of methods of analytical, semianalytical or numerical integration, etc.

In this paper, following R.S. Harrington, we have operated with the equations of motion in their Hamiltonian form, while their integration was approached by using the perturbation theory of Deprit (1969), in which the application of the Lie transformation to the initial canonical system gives canonical systems more amenable to integration. There is no unique way to apply this method, since the angular variables that appear in the Hamiltonian function may be eliminated with a single canonical transformation, or one by one using two or three transformations. The constants of integration may, furthermore, be recalculated at fixed time intervals or those obtained initially can be used throughout the process.

Experience has shown us that the results obtained using these forms of applying the method are in close agreement, therefore we decided to jointly eliminate the angular variables and recalculate the constants of integration at intervals of time coinciding with the non-perturbed period of the inner orbit. We have considered up to the third-order term in the small parameter in powers from which the Hamiltonian function is developed; that is, we have only used the term that most contributes to the perturbations of both orbits.

The software obtained, which we call Program Pertur, is here applied to the well-known systems $\xi$ Ursae Majoris (ADS 8119 - WDS $11182 \mathrm{~N} 3133$ ) and $\zeta$ Aquarii (ADS 15971 - WDS 22288S 0002).

\section{APLICATION TO $\xi$ URSAE MAJORIS}

This system is really a quadruple star formed of two subsystems $A a$ and $B b$. Stars $B$ and $b$ form a spectroscopic binary of period 3.98 days and so, to a 
first approximation, this subsystem can be considered as a single star, the total system becoming a triple star with two close members $A$ and $a$, and a distant member $B b$.

$\xi$ Ursae Majoris is probably the system which best satisfies the requirements to be able to study perturbations in its motion. Because of this, it was used in earlier studies, chief among these being those of Brown (1936-37), Heintz (1967), Harrington (1968a), and Ling (1989).

In order to apply our theory to this star we have used the elements and masses published by W.D. Heintz to initialize the calculation process.

\subsection{Results}

The results obtained are shown in Tables 1 and 2 , in which we give the values of the orbital elements calculated $25,50,75$ and 100 years after the initial moment $t_{0}=1900.0$. (Masses: $\left.m_{A}=0.83 \mathcal{M}_{\odot}, m_{a}=0.31 \mathcal{M}_{\odot}, m_{B b}=0.92 \mathcal{M}_{\odot}\right)$.

TABLE 1. Inner orbit

\begin{tabular}{|c|c|c|c|c|c|c|c|}
\hline$t-t_{0}$ & $P_{1}$ & $T_{1}$ & $c_{1}$ & $a_{1}$ & $i_{1}$ & $\Omega_{1}$ & $\omega_{1}$ \\
\hline $0^{y}$ & $1^{y} 832$ & 1935.41 & 0.560 & $0^{\prime \prime} .202$ & $83^{\circ} .6$ & $326^{\circ} .0$ & $146^{\circ} .0$ \\
\hline $25^{y}$ & $1^{y} 833$ & 1936.748 & 0.558 & $0^{\prime \prime} .202$ & $86^{\circ} .27$ & $323^{\circ} .86$ & $143^{\circ} .05$ \\
\hline $50^{y}$ & $1^{y} 827$ & 1934.477 & 0.560 & $0^{\prime \prime} .201$ & $88^{\circ} .55$ & $321^{\circ} .63$ & $140^{\circ} .53$ \\
\hline $75^{y}$ & $1^{y} 827$ & 1935.799 & 0.555 & $0^{\prime \prime} .202$ & $91^{\circ} .13$ & $319^{\circ} .36$ & $138^{\circ} .16$ \\
\hline $100^{y}$ & $1^{y} 827$ & 1935.242 & 0.554 & $0^{\prime \prime} .202$ & $94^{\circ} .03$ & $316^{\circ} .24$ & $135^{\circ} .02$ \\
\hline
\end{tabular}

\section{APLICATION TO $\zeta$ AQUARII}

This triple star was previously studied by R. S. Harrington (1968b) and W. D. Heintz (1984). This system closely resembles the previous one except that the orbital periods are much longer (about 10 times), therefore the perturbations are observed with difficulty in the aproximately 200 years for wich there are observations.

In this case the elements used to initialize the calculation process were those published by Heintz in 1984. The variations of the elements with secular perturbations in 10 centuries were:

$$
\begin{aligned}
& \Delta i_{1}=14^{\circ} .3 \quad \Delta i_{2}=-1.2 \\
& \Delta \Omega_{1}=-10^{\circ} .2 \quad \Delta \Omega_{2}=0^{\circ} .9 \\
& \Delta \omega_{1}=-5^{\circ} .0 \quad \Delta \omega_{2}=0^{\circ} .7
\end{aligned}
$$


TABLE 2. Outer orbit

\begin{tabular}{|c|c|c|c|c|c|c|c|}
\hline$t-t_{0}$ & $P_{2}$ & $T_{2}$ & $e_{2}$ & $a_{2}$ & $i_{2}$ & $\Omega_{2}$ & $\omega_{2}$ \\
\hline $0^{y}$ & $59^{y} 84$ & 1935.17 & 0.414 & $2^{\prime \prime} .53$ & $122^{\circ} .65$ & $101^{\circ} .59$ & $127^{\circ} .53$ \\
\hline $25^{y}$ & $59^{y} 85$ & 1935.64 & 0.414 & $2^{\prime \prime} .53$ & $122^{\circ} .35$ & $101^{\circ} .54$ & $127^{\circ} .90$ \\
\hline $50^{y}$ & $59^{y} 887$ & 1936.08 & 0.415 & $2^{\prime \prime} .531$ & $122^{\circ} .15$ & $101^{\circ} .30$ & $127^{\circ} .84$ \\
\hline $75^{y}$ & $59^{y} 911$ & 1936.54 & 0.414 & $2^{\prime \prime} .532$ & $121^{\circ} .80$ & $101^{\circ} .30$ & $127^{\circ} .66$ \\
\hline $100^{y}$ & $59^{y} 912$ & 1937.16 & 0.415 & $2^{\prime \prime} .533$ & $121^{\circ} .58$ & $100^{\circ} .94$ & $127^{\circ} .32$ \\
\hline
\end{tabular}

It is forseeable that high resolution techniques, such as speckle interferometry, permit the discovery of new triple systems of the $\xi$ Ursae Majoris type or even faster which will facilitate the comparison of the different approaches to the stellar three-body problem.

\section{REFERENCES}

Abad, A.J. 1984, Estudio de Sistemas Estelares Múltiples. Pub. Seminario García Galdeano, Ser. II, Sec. 2, num. 2, Universidad de Zaragoza. Spain

Brown, E.W. 1936-37, The Stellar Problem of Three Bodies, MNRAS, 97, num. 7, 25 Deprit, A. 1969, Canonical Transformations Depending on a Small Parameter, Celes. Mech., 1, 12

Docobo, J.A. 1977, Aplicación de la teoría de Perturbaciones al estudio de sistemes estelares triples. Tesis Doctoral. Universidad de Zaragoza

Docobo, J.A. \& Prieto, C. 1988, The Stellar Three-body Problem. Explicit Formulation for Computing Perturbations. Pub. Obs. Ast. R.M. Aller, 46

Harrington, R.S. 1968a, Dynamical Evolution of Triple Stars, AJ, 73, 190

Harrington, R.S. 1968b, Dynamics of the Triple System Zeta Aquarii, AJ, 73, 508

Harrington, R.S. 1969, The Stellar Three-body Problem, Celes. Mech., 2, 200

Heintz, W.D. 1967, Storungen und Bahnelemente im System $\xi$ Ursae Majoris, Astrom. Nach., 289-6, 269

Heintz, W.D. 1984, The Triple Star Zeta Aquarii, ApJ, 284, 806

Ling, J.F. 1989, Aplicación del método estroboscópico al problema estelar de tres cuerpos. Tesis Doctoral. Universidad de Santiago 